\title{
A Model Based Toolchain for the Cosimulation of Cyber-Physical Systems with FMI
}

\author{
David Oudart $^{1,2}$, Jérôme Cantenot ${ }^{1}$, Frédéric Boulanger ${ }^{3}$ and Sophie Chabridon ${ }^{2}$ \\ ${ }^{1}$ EDF RED, Palaiseau, France \\ ${ }^{2}$ SAMOVAR, CNRS, Télécom SudParis, Institut Polytechnique de Paris, France \\ ${ }^{3}$ LRI, CNRS, CentraleSupélec, Université Paris-Saclay, France
}

Keywords:
Cosimulation, FMI, IT, MDE, smart grid, cyber-physical system

Abstract:

Smart Grids are cyber-physical systems that interface power grids with information and communication technologies in order to monitor them, automate decision making and balance production and consumption. Cosimulation with the Functional Mock-up Interface standard allows the exploration of the behavior of such complex systems by coordinating simulation units that correspond to the grid part, the communication network and the information system. However, FMI has limitations when it comes to cyber-physical system simulation, particularly because discrete-event signals exchanged by cyber components are not well supported. In addition, industrial projects involve several teams with different skills and methods that work in parallel to produce all the models required by the simulation, which increases the risk of inconsistency between models. This article presents a way to exchange discrete-event signals between FMI artifacts, which complies with the current 2.0 version of the standard. We developed a DSL and a model-based toolchain to generate the artifacts that are necessary to run the cosimulation of the whole system, and to detect potential inconsistencies between models. The approach is illustrated by the use case of an islanded grid implementing diesel and renewable sources, battery storage and intelligent control of the production.

\section{INTRODUCTION}

Strong and fast evolutions of the electric landscape deeply impact the existing power grids. Electric vehicles, decentralized and renewable production as well as political considerations require the grid operators to continuously adapt their operating strategy and the grid equipments. Smart Grid is the name given to the oncoming generation of power grids, augmented with information and communication technologies. Such systems aim to allow prevention, better reactivity and improved response to electrical events such as failures, but also ensure proper interoperability between all the components of the grid. Because they may impact a large amount of people, smart grids are critical systems so they require thorough verification and validation before their implementation. To this end, simulation is very valuable to evaluate various behavioral assumptions (Palensky et al., 2017).
As they involve many interdependent technical domains, namely electronics, software information processing and telecommunications, smart grids are a typical example of CyberPhysical System (CPS), with the same challenges to face when it comes to modeling and simulation. Designing a CPS usually involves several teams working simultaneously on modeling multiple aspects of the system. Interconnecting these models implies to deal with heterogeneous models of computation, data consistency issues, discrete and continuous variabilities, and various time-steps (Muller et al., 2018). The lack of mature smart grid simulation tools lead Rohjans et al (Rohjans et al., 2014) to establish a list of requirements for an appropriate tool addressing these challenges.

In addition, industrial companies are reluctant to changes in their work methods and tools, and prefer to rely on the existing skills and strengths of their teams. Simulating CPS amounts to find 
ways and tools to model and simulate interactions between discrete and continuous models, taking the most from the existing skills of the industrial teams who are developing these systems.

Cosimulation approaches allow each one of the teams involved in the modeling phase to use their own language and tool of predilection, as well as the most suited paradigm for their domain. There are several standards for cosimulation, and the Functional Mockup Interface (FMI) ${ }^{1}$, is particularly suited to industrial projects.

Our main objective is to provide a toolchain to help modeling teams to create interoperable models. For that purpose, we developed a Domain Specific Language (DSL) and we rely on $\mathrm{MDE}$ practices to automatically generate wrappers, interfaces and deployment scripts compliant with the FMI standard, and handling the continuousdiscrete interconnections. This minimizes the effort required from the model developers to adapt the models, and reduces the risk of errors and the deployment cost of each iteration.

In the next section, we explain why we chose FMI for the cosimulation of smart grids and the limitations of this choice. In Section 3, we propose a way to deal with the limitations of FMI for discrete signals. Section 4 presents our toolchain to build the simulation of a CPS, dealing with the industrial and FMI constraints. Section 5 illustrates the approach on a use case. Section 6 presents scenarios to validate our approach.

\section{STATE OF THE ART}

In the electrical energy community, the challenge of simulating smart grids is not new ( $\mathrm{Li}$ et al., 2011, Nutaro, 2011, Yang et al., 2013). However, it usually consists in the interaction of two domains via two dynamic models. The problem of the synchronization of models and of their consistency is not specifically addressed by these approaches, but is not really challenging when limited to two domains.

For the industrial simulation of complex systems and CPS, it is better to rely on standard technologies, as they address various needs like scalability, modularity or reusability. The Functional Mockup Interface (FMI) (Blochwitz et al., 2011) and The High Level Architecture (HLA) (Dahmann and Morse, 1998) are two interoperability approaches allowing the intercon-

\footnotetext{
${ }^{1}$ https://fmi-standard.org
}

nection of several different simulators in an integrated execution.

If both approaches have been declared as standard, FMI benefits from a stronger popularity with more than 80 compatible tools $^{2}$. Its ability to protect industrial property inside FMUs makes it very attractive for industrial projects and makes collaborative design easier (Gomes et al., 2018b). FMI defines a simulation unit format called Functional Mockup Unit (FMU), which embeds a model and an execution engine along a standard interface to control the execution of the simulation. FMI cosimulations are driven by a master algorithm, which synchronizes the execution of the FMUs and the exchange of data at some communication points.

Because a time-step between two communication points can not be null, FMI is not particularly adapted to reactive systems and discreteevent modeling. Current works already propose FMI extensions, such as zero-size steps (Guermazi et al., 2015, Cremona et al., 2019), or absent values (Cremona et al., 2019) to handle discrete-event signals. Optimized master algorithms (Tavella et al., 2016, Van Acker et al., 2015) can increase the precision of the simulation while still being compliant with the standard, by trying to locate the occurrence of an event using the optional rollback FMI feature (reverting the state of an FMU to a previous communication point), or by optimizing the choice of the time step, which requires FMUs to be exported as white boxes.

Moreover, connections between FMUs are defined statically before execution, meaning that every possible interaction between models has to be defined during the modeling phase and the cosimulation set up. This can lead to many revisions and iterations on the models while trying to interoperate them with the other models, and potentially generate a lot of input/output ports to cover all possible scenarios. Adaptation approaches (Gomes et al., 2018a) allow the reuse of existing models or FMUs by wrapping them in a simulation unit adapted to a particular cosimulation.

This last issue is derived from the fact that complex system design involves several viewpoints from different technical domains, therefore several heterogeneous models developed by different teams. Model driven approaches are used in industry to interconnect these models and represent the consistency links between them (Zhao

\footnotetext{
${ }^{2}$ https://fmi-standard/tools/
} 
et al., 2017, Suri et al., 2017, Andrén et al., 2017). These approaches mainly aim to facilitate the final system realisation, but only few of them include the simulation in the design process (Paris et al., 2017).

FMI offers a technological means to simulate a CPS such as a smart grid, but requires a supportive method and/or tool to address the various issues raised. The toolchain we developed supports model transformations and focuses on the deployment of a correct cosimulation platform. It complies with the FMI standard and relies on existing tools which implement it, but also supports exchange of discrete-event signals, as smart grids involve cyber components. It provides a domain specific language to allow the modeling teams to identify and characterize all signals exchanged between FMUs in a cosimulation. Finally, it automates the generation of simulation artifacts implementing these interfaces, such as configuration files, execution scripts and model adaptators.

\section{HOW TO EXCHANGE DISCRETE SIGNALS BETWEEN MODELS VIA FMI}

FMI is designed for the interconnection of simulators producing continuous signals. Therefore, for simulations involving information processing and communication networks, which rely on discrete events, we need to encode such sporadic events using continuous signals.

\subsection{Problem and Definitions}

We suppose the set $\mathcal{V}$ of all the values a signal can take is one of the following sets: $\mathbb{R}, \mathbb{Z}, \mathbb{S}$ the set of all string values, or $\mathbb{B}$ the set of all boolean values $\{$ true, false $\}$.

A discrete-event signal can then be represented as a function of time $x_{D E}: \mathcal{E} \rightarrow \mathcal{V}$ where $\mathcal{E} \subset \mathbb{R}^{+}$is the discrete set of the event-firing points.

The problem is that signals in an FMI cosimulation are only considered at some communication points chosen by the master. The set $C$ of the communication points being independent of the set $\mathcal{E}$, if we want to exchange a discrete event as an FMI signal, we have a risk of undefined or missed events:

- $\mathcal{E} \cap \mathcal{C} \subset \mathcal{C}$ : at some communication point, the value of the signal is not defined. (pb. 1)
- $\mathcal{E} \cap \mathcal{C} \subset \mathcal{E}$ : some events are missed by the master of the cosimulation and the other FMUs (pb. 2)

The three possible cases are illustrated in Figure 1.

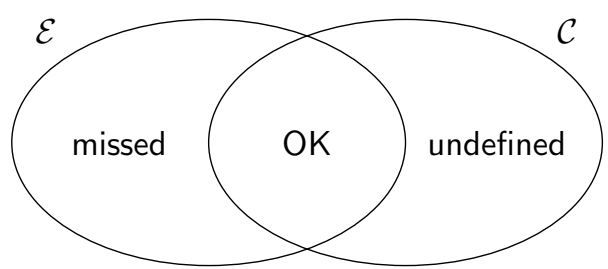

Figure 1: Differents cases for discrete event signals

\subsection{Proposed Solution}

In order to be sure that the exchanged signal is defined on every communication points and avoid pb. 1, we need an FMU to produce a time-continuous signal defined on $\mathbb{R}^{+}$(at least bounded by the start and stop times of the cosimulation).

We propose an encoding component to transform a discrete signal into two timecontinuous signals which can be exchanged over FMI. The first time-continuous signal keeps the value of the last event emitted until a new event is emitted. But we need a second coupled signal to express that an event actually has been emitted, in order to detect sequences of events having the same value. Indeed, each time the value of our first signal changes we know that an event has occurred, but while it is constant we cannot detect if there has been an event with the same value or no event.

Following these principles, we also provide a decoding component to perform the reverse operation and obtain the discrete-event signal from the FMI discretization of the timecontinuous signals.

\subsubsection{Encoding Component}

Input: $x_{D E}$ a discrete-event signal. Output: $x$ and $x_{s}$ two time-continuous signals.

We consider $\mathcal{E}=\left\{t e_{1}, t e_{2}, \ldots\right\}$ as ordered.

$$
\forall t \in \mathbb{R}^{*}, t \geq t e_{1} \quad\left\{\begin{array}{l}
x(t)=x_{D E}\left(t e_{n_{t}}\right) \\
x_{s}(t)=n_{t}
\end{array}\right.
$$

where $n_{t}=\max \left\{i \mid t e_{i} \leq t\right\}$ is the index of the last event that occurred before or at time $t$.

When $t<t e_{1}$, no event has been emitted yet, and $x_{s}(t)=0 . \quad x(t)$ can have any value, which 
will be ignored by the decoding component. We suggest to choose an initial value corresponding to the last event which could have occurred before the start of the simulation. When it is not possible or does not make sense, a default value in the $\mathcal{V}$ set $(0$, an empty string or false) is a valid choice too.

\subsubsection{Decoding Component}

Input: $y$ and $y_{s}$ two time-continuous signals sampled over $\mathcal{C}$. Output: $y_{D E}$ a discrete-event signal.

$\mathcal{C}=\left\{t c_{0}, t c_{1}, t c_{2}, \ldots\right\}$ is the set of the communication points. We assume the previous definition of the set $\mathcal{E}$, and the fact that $y$ and $y_{s}$ are the $x$ and $x_{s}$ signals discretized by the FMI cosimulation master on $\mathcal{C}$.

We define the discrete set $\mathcal{F} \subset \mathbb{R}^{+}$as the set of the time points of the $y_{s}$ 's "rising edges". These time points are all synchronized with a communication point so we have:

$$
\mathcal{F}=\left\{\begin{array}{l|l}
t c_{i} \in \mathcal{C} & \begin{array}{l}
i>1, y_{s}\left(t c_{i}\right)>y_{s}\left(t c_{i-1}\right) \\
i=0, y_{s}\left(t c_{0}\right) \neq 0
\end{array}
\end{array}\right\}
$$

Then the output of our component is:

$$
\begin{gathered}
y_{D E}: \mathcal{F} \rightarrow \mathcal{V} \\
\forall i, t c_{i} \in \mathcal{F} \subset \mathcal{C} \quad y_{D E}\left(t c_{i}\right)=y\left(t c_{i}\right)=x_{D E}\left(t e_{n_{t c_{i}}}\right)
\end{gathered}
$$
where $n_{t c_{i}}$ and $t e_{n_{t c_{i}}} \in \mathcal{E}$ are defined as before as the index of the last event occurring before or at time $t c_{i}$, and the time of this event.

\subsection{Discussion}

We are aware that several challenges still need to be addressed in order to allow discrete-event signal exchanges over FMI. The dates of the events are almost always approximated from the global cosimulation perspective. It prevents a rigorous handling of concurrent events for instance. For this issue, we are limited by the definition of time in the FMI standard (Broman et al., 2015). Our goal is to allow a better support of the discreteevent signals with a minimal adaptation effort.

Using our two components to encode and decode a discrete-event signal between two FMUs, and using the same notation as previously, an event received at $t c_{i}$ has been emitted at $t e_{n_{t c_{i}}}$. We know that $t e_{n_{t c_{i}}}$ is the latest time point of $\mathcal{E}$ such as $t c_{i-1}<t e_{n_{t c_{i}}} \leq t c_{i}$. So our solution addresses pb. $\mathbf{2}$ by ensuring that if an event is not synchronized with a communication point, it can still be detected, but delayed by $t c_{i}-t e_{n_{t c_{i}}}$. This

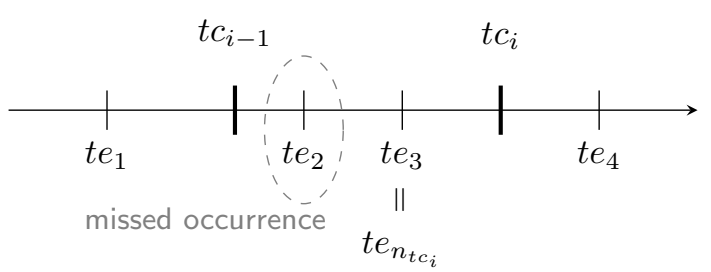

Figure 2: Missing events that are too close

is an inherent limitation of the current version 2.0 of FMI.

However, pb. $\mathbf{2}$ is not completely addressed, as events emitted between $t c_{i-1}$ and $t e_{n_{t c_{i}}}$ are still missed as shown in Figure 2. We can only express at most one event at each communication point. It is an arbitrary choice by only considering the last emitted one in the time-step interval. Nonetheless, the difference between $y_{s}\left(t c_{i}\right)$ and $y_{s}\left(t c_{i-1}\right)$ still reveals the number of events emitted since the last communication point. A smart-enough cosimulation master might decide to cancel the last step and replay it with a smaller step in order to catch the missed events. This requires the optional rollback functionality of the FMI standard.

Finally, the use of these components in the FMU models requires potentially heavy changes to the original models, as well as the addition of FMI connections dedicated to the synchronization of FMUs. This kind of knowledge is specific to the cosimulation process and does not need to be the concern of the modeling teams.

The toolchain presented in Section 4 provides methods and tools to generate these components for the modeling teams, and to automate the deployment and the possible iterations of the smart grid cosimulation model.

\section{TOOLCHAIN PRESENTATION}

\subsection{An Approach Based on Model Refinement}

One of the main advantages of using a cosimulation environment is to allow the different experts to develop their own model in autonomy, with a minimal interference and in parallel with the others (Gomes et al., 2018b). The choice of the FMI standard ensures the technological compatibility of each simulation unit, or FMU, with the 
cosimulation environment, without having to develop a specific connector. However it does not ensure structural compatibility. All FMUs produced by the different teams must provide interoperable data structures, namely each input should match an output, in type and meaning.

An example of a cosimulation approach for smart grids (Oudart et al., 2019) identified several steps and actors involved in such a process. The first step is to define all the connections between the simulation models in order to define the interface of the models for each modeling team. But the compliance verification of the models and the creation of the cosimulation artifacts (FMUs, configuration files) are done by hand, which make each iteration time-consuming and error-prone.

The use of a global, architectural model to represent the structural interfaces of the various simulation units and the coupling constraints between them, allows the use of syntactic tools to automatically check some validation rules. It also creates a unique authoritative artifact to coordinate the work of the various collaborators, and from which more detailed models can be derived.

Following this approach, we developed a toolchain to automate the actions needed to run a cosimulation starting from a global model. It is based on model transformations from a platformindependent model toward simulation artifacts. This toolchain relies on a domain specific language (DSL), named Cosimulation Modeling Language (CosiML), to specify the structural interfaces of the simulation units and the configuration of the cosimulation.

Our choice to develop our own language for this purpose, instead of choosing an existing one, such as UML, comes from various reasons :

- in an industrial context, general-purpose languages like UML are not well mastered outside the computer science field,

- such languages contain many concepts, but we only needed a few of them,

- in our approach, adapting UML to model specific concepts would lead to refining generic concepts through profiles.

It appeared more efficient to define only what we needed than to restrict and specialize UML to fit our needs.

\subsection{CosiML, a DSL for Cosimulation}

We implemented CosiML inside the Eclipse Modeling Framework (EMF) using the Ecore metamodeling language. Figure 3 shows a simplified metamodel of CosiML, with the classical elements of every cosimulation.

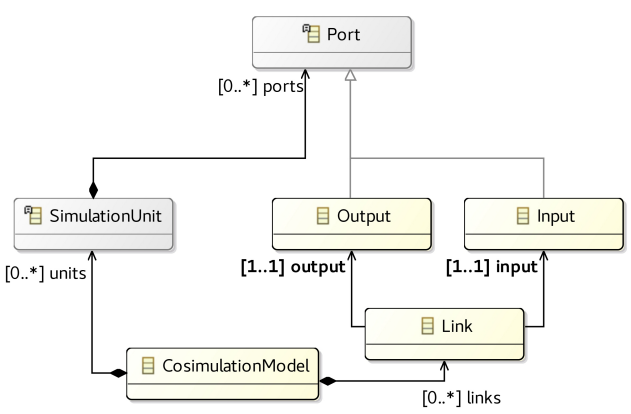

Figure 3: CosiML simplified metamodel

CosimulationModel is the root element of the model, it stores the parameters of the cosimulation (start time, stop time, time step, etc.) and contains all the simulation units and their interconnections.

SimulationUnit represents a simulation unit involved in the cosimulation. It contains the Port elements representing the structural interface of the unit.

Input \& Output (Port) represents a port of the simulation unit. It has a type, an optional default value and a variability, which is the name used by FMI to characterize the discrete or continuous nature of signals.

Link represents a connection between an output and an input port. A model can be checked to verify that any two connected ports have the same variability, and that they are not contained in the same simulation unit.

There are several kinds of SimulationUnits, as shown in Figure 4:

ProvidedSimulationUnit is a simulation unit which is completely provided by the user. Such a simulation unit is directly usable in the cosimulation without further action. In our case of FMI cosimulation, a ProvidedSimulationUnit is provided as an FMU resource and we only have to know the path to the artifact.

GeneratedSimulationUnit is a simulation unit which will be generated by the toolchain from a domain model. The attribute modelPath stores the path to the domain model. The format 


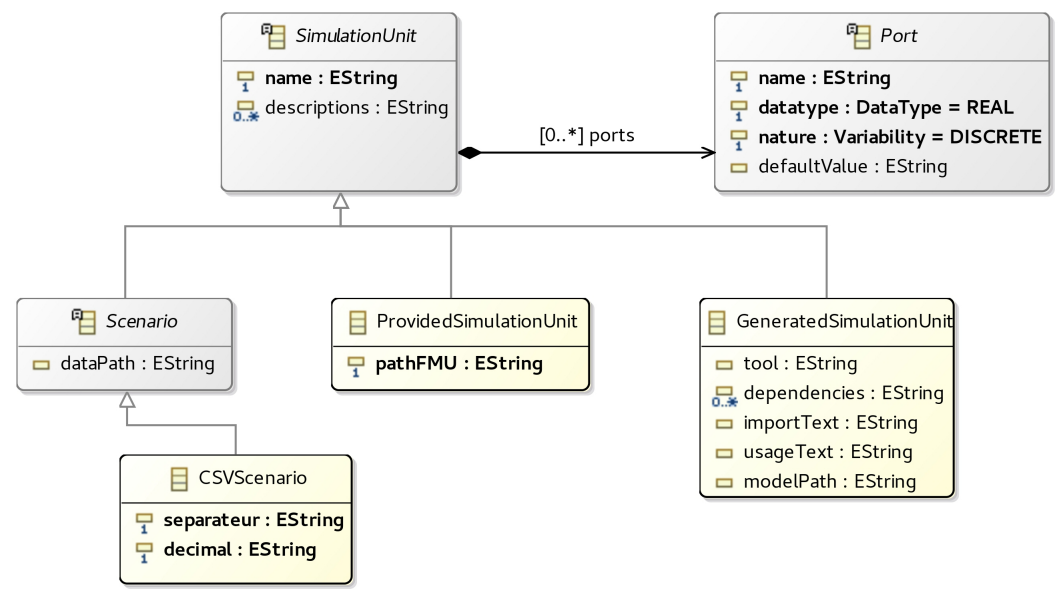

Figure 4: SimulationUnit detailed specializations

of the model and the generator to use for the generation of the simulation unit are specific to the tool attribute's value. The tool is what is used to build the model, for instance a Java or $\mathrm{C}++$ compiler, or a more complex modeling tool such as OMNeT for communication networks. The generator is part of our toolchain, and will generate the corresponding FMU, which includes the generation of adapters for discrete event signals. The generator relies on naming conventions to access the elements of the model and adapt them to the structure of the FMU. For instance, a Java with a continuous input signal named $\mathrm{X}$ should implement a setX (double value) method. In order to refer to the model in the generated FMU, the generator uses two generic attributes: importText defines how to import the model inside the adapter, and usageText tells how to use the model. Finally, the attribute dependencies stores the list of all the resource paths required by the model (libraries, data files, binaries) that should be packaged inside the generated simulation unit. Our goal is to stay generic enough to avoid metamodel modifications when we want to support a new tool and add a new generator to the toolchain. For instance, a Java model-based generator would require:

importText $=$ import package. Classname; and

usageText $=\mathrm{Cl}$ assname,

whereas a $\mathrm{C}++$ model-based generator would require:

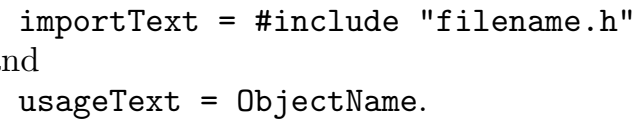

Scenario a simulation unit generated by the toolchain from a data file. It only has output ports and will be used as an independent source of timed data. The attribute dataPath stores the path to the data file. We are considering that future versions of CosiML and the toolchain may support several format, but for now we only support CSV files to be used as Scenario units.

CSVScenario a particular Scenario element which refers to a CSV data file. Attributes separator and decimal defines the characters used respectively as separator and decimal marker for the CSV content.

\subsection{Generation Tools for FMI Cosimulation}

We chose the DACCOSIM NG ${ }^{3}$ software to execute our FMI cosimulation. It implements a master algorithm that is fully compliant with the standard, with advanced discontinuity detection features, and intelligent time step strategies (Tavella et al., 2016). More importantly, it provides a scripting language allowing the automation of the build and execution of cosimulations. Finally it is designed for distributed executions, which is very useful for industrial use cases potentially involving a large number of FMUs (Évora Gómez et al., 2019).

With our CosiML metamodel defined in EMF Ecore, we can use the Sample Reflective Ecore Model Editor to instantiate a CosiML model and serialize it in the XMI format. Then, we can use model transformations to implement our toolchain. We developed an Acceleo plugin to ge-

\footnotetext{
${ }^{3}$ https://bitbucket.org/simulage/daccosim
} 
nerate all the files needed to build the FMI cosimulation from the CosiML model. The Figure 5 shows the generation process of these files from a CosiML model. The generators are configured with property files, used to specify platform dependent information, such as library and tool paths.

The toolchain is currently composed of the following generators :

1. Java-tool generator: generates all the files needed to build an FMU from a Java model. It is applied to the GeneratedSimulationUnit instances with the right tool property. It generates a Java file defining a class adapting the user model to the JavaFMI library ${ }^{4}$, along with a MANIFEST.MF file defining the proper classpath. It also generates a script to build the corresponding FMU.

2. CSV scenario generator: generates the files needed to build an FMU from a CSV file. It is applied to the CSVScenario instances. It generates a Java file defining a class loading the CSV file, and implementing the JavaFMI library, along with the MANIFEST.MF file and the building script, just as with the Javatool generator.

3. Cosimulation scripts generator: generates the DACCOSIM cosimulation model in its specific scripting language DNG. It also generates an execution script, which automates the build of all the FMU not yet generated, and the launch of the DACCOSIM simulation.

CosiML allows the distinction between discrete and continuous data exchanges, so that the provided generators can automatically implement the encoding and decoding components in the generated wrappers, and adapt the FMU inputs and outputs accordingly (each CosiML Port with a discrete variability causes the creation of two FMI ports). Our toolchain is meant to be extended with other generators to support more domain specific tools and to be used for cyberphysical systems other than smart grids. The next section presents an industrial use case of smart grid cosimulation, and shows the integration of the current toolchain with the usual modeling tools of the different teams.

\footnotetext{
${ }^{4}$ bitbucket.org/siani/javafmi/, a set of component to work with FMI. It especially provides a builder component generating an FMU from Java code
}

\subsection{Download}

Our toolchain is shared on a github repository at:

https://github.com/davidoudart-pro/SGridSF

The sources of the CosiML language and generation plugins are available, as well as the necessary files to replay the cosimulation of the use case presented in this article.

\section{USE-CASE COSIMULATION}

\subsection{The Use-Case of an Islanded Smart Grid}

We chose a real use case from the French power utility to illustrate our contribution, and validate our toolchain. The system is an island with a power grid that is independent from the mainland grid, with its own production equipments. A diesel power plant is the main energy producer, and is complemented by a photovoltaic farm. The main issue in the configuration is that the renewable energy supply is intermittent. Indeed, as the photovoltaic source relies on sunlight and needs a clear sky for its production, it makes it as variable and unpredictable as the weather. In order to balance the production with the consumption, it has to be sometimes prevented from producing as much as it could, which causes economic loss and carbon footprint degradation. Therefore, a chosen solution is to add a battery storage to damp the variability of the production, with the purpose of minimizing the limitations of the photovoltaic farm. It could even allow the operator to shut down the diesel plant for some period and rely only on the battery and photovoltaic production.

To maximize the efficiency of the system, we need an Energy Management System (EMS) coupled with a Supervisory Control And Data Acquisition (SCADA) in order to implement an intelligent control of the production. The EMS monitors the state of the power grid (value of the voltage at various control points, state of switches), and drives some of its equipments (giving voltage setpoints, limiting the injection of power by a source) through the SCADA. The EMS can collect other information such as weather and consumption forecasts from external information systems, as well as user preferences, in order to optimize the operation of the grid. 


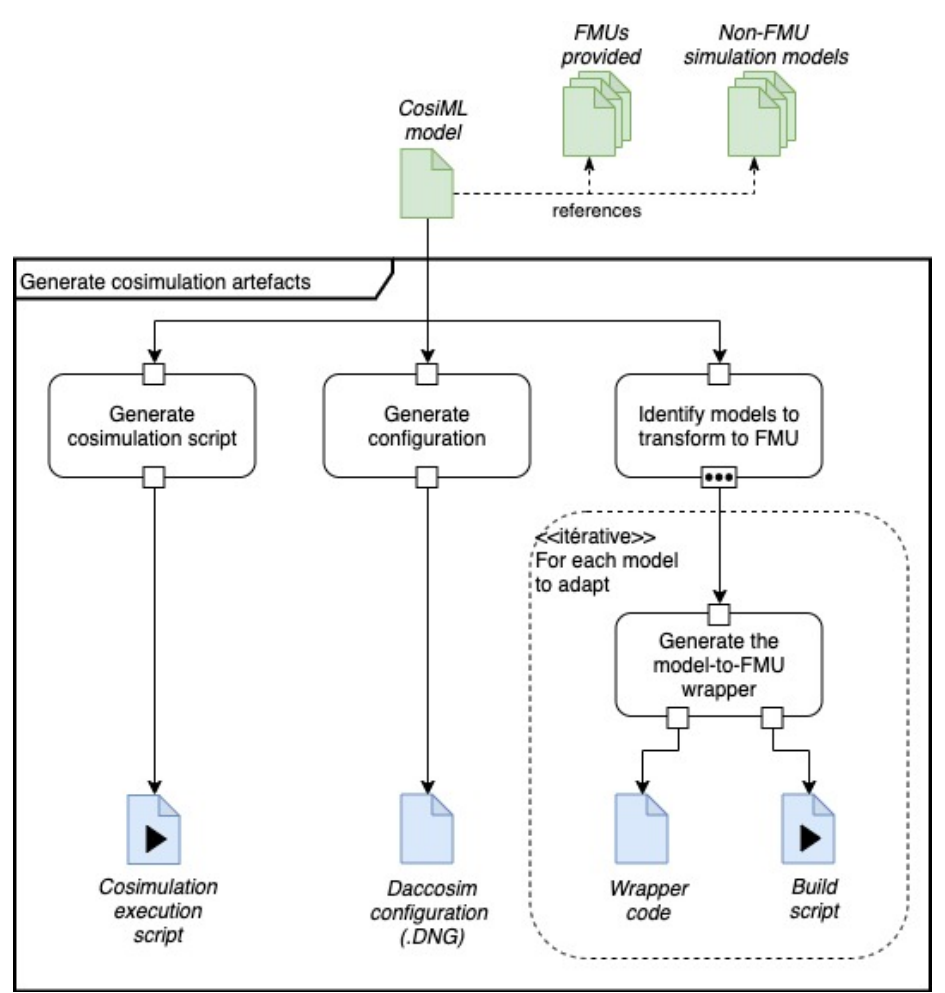

Figure 5: Generation process of the cosimulation artifacts, from a CosiML model

Before telling how the EMS controls the equipments on the grid, we have to explain how the power flow is established on a power grid. Knowing the power needed by the consumers, we can set power production setpoints to the various sources of the grid in order to balance the consumption. However, losses on transmission lines can never be known, so we need at least one equipment not power constrained, capable of producing the loss or absorb the unpredictable excess. This equipment is generally the one having the highest size generator. In our case it is the diesel plant when it is connected to the grid, the battery and its converter when it is not.

The EMS sends control signals to the various equipment of the grid:

- photovoltaic farm: the EMS decides if the production needs to be limited and how much;

- battery: there are two cases for this equipment. When the diesel plant is coupled to the grid and balances the power on the grid, the EMS controls the power absorbed or injected by the battery. When the diesel plant is shut down, the EMS does not control the battery and lets its power converter balance the power on the grid.

- diesel plant: the EMS decides if it is cou- pled to the grid (and produces power) or not. When it is coupled to the grid, it cannot produce less than a minimum power, so it can happen that the photovoltaic farm has its production limited. To avoid it, the diesel is turned off when the battery and the photovoltaic production are able to cover the consumption needs.

Because of all the different modes in which the grid can be, depending on the weather, on the management of the charge of the battery and on the variability of the consumption, simulation is very useful to test and validate a design of the solution, before any deployment on the field and expensive investments.

\subsection{Cosimulation Scenario}

The simulation of the islanded smart grid is a good example of a cyber-physical system involving several knowledge fields, and several teams with different modeling tools. We used Modelica ${ }^{5}$ with the Dymola software to model the grid power flow because they are well-known tools among electrical engineers, and they fully support the

\footnotetext{
${ }^{5}$ https://www.modelica.org/, component-oriented modeling language based on equations set declaration
} 
FMI standard and the export to FMU (Elsheikh et al., 2013). The simulation unit continuously evaluates the electrical power state of the grid according to production and consumption constraints. Figure 6 shows the simplified model of the power flow and its inputs.

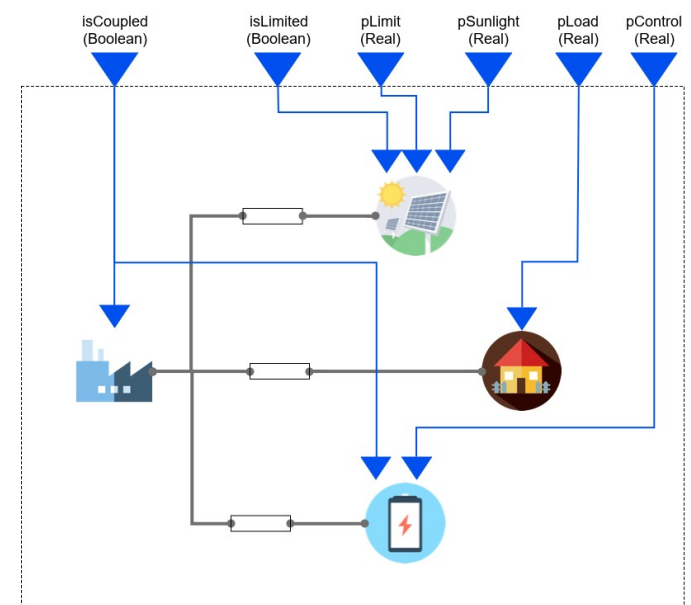

Figure 6: Simplified Modelica model of the islanded grid

Some of these constraints are computed and decided by the algorithm of the EMS. The EMS produces discrete event signals, reacting to the evolution of the state of the grid. There is no conventional tool supporting the modeling of reactive systems and also handling FMI. Complex algorithms are usually modeled with textual procedural languages such as $\mathrm{C}$ or Java. There are tools supporting the export of such models toward FMU, but they require additional efforts and specific code refactoring and writing. Our toolchain supports the automatic transformation of a Java model into an FMU, with the generation of a wrapper code implementing the JavaFMI Framework library, and the use of the JavaFMI builder tool. We developed a first, simple Java algorithm of the EMS which takes the current state of the grid as input and does not use forecasts. It computes controls every 15 minutes, but continuously monitors the current state of the grid equipments in case emergency controls are required. The Figure 7 shows an activity diagram, illustrating this process. This simple model can give reference simulation results, as a base of comparison with more complex and complete algorithms provided by various vendors.

These components need to be tested on multiple scenarios. We have access to some data sets describing the consumption of the island and the weather conditions over time at various times of

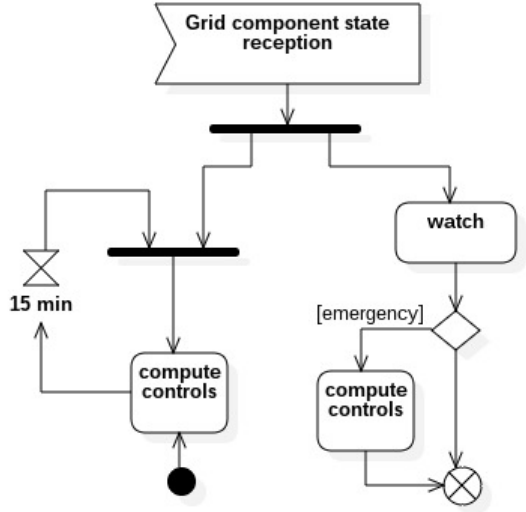

Figure 7: EMS monitoring process, with periodic and emergency controls

the year. The toolchain also supports the automatic transformation of a timed-CSV file into an FMU, so we can use these data sets in our cosimulation (via the CosiML CSVScenario element).

Eventually, our CosiML cosimulation model involves three simulation units:

- GridFmu which computes the electrical state of the system

- EmsFmu which computes the controls to send to the grid components

- CurvesFmu which plays the consumption and sunlight intensity evolution from the given data sets.

The CosiML model of this cosimulation instantiates GridFmu as a ProvidedSimulationUnit, because it is provided as an export from Dymola. EmsFmu is instantiated as a GeneratedSimulation Unit because the model is provided as java source code, and our toolchain will generate the FMU. Finally CurvesFmu is instantiated as a $C S V S c e n a r i o$, because we are provided with a CSV file that will be used to generate an FMU that plays the data contained in the file.

As we said previously, the CosiML model has been edited with the EMF's Sample Reflective Ecore Model Editor, and stored in the XMI format. Figure 8 illustrates the XMI CosiML model's content by expliciting the connections between the three FMUs and their variability (solid lines for continuous signals, dotted lines for discrete events).

\subsection{Simulation and Decisions}

The use case presents two main concerns: 1) how to optimize the battery characteristics in order 


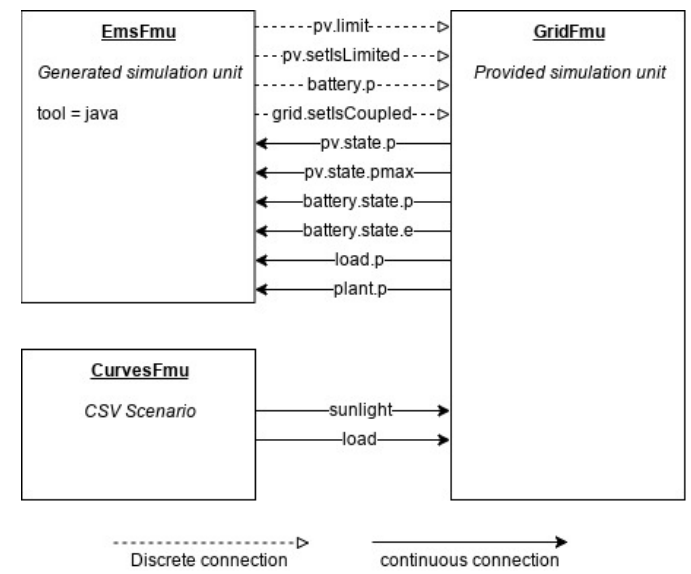

Figure 8: Connections between the FMUs of the use case

to implement an efficient management of the production and keep investment as low as possible? And 2) how to test the efficiency of the chosen EMS algorithm?

From the CosiML model, the toolchain generates the necessary wrapper files to build the EmsFmu and CurvesFmu FMUs, as well as the DACCOSIM model of the cosimulation. In addition, a script is generated to create automatically the missing FMUs, and to launch the DACCOSIM cosimulation.

In our case, the cosimulation evaluates the behavior of the grid on a full day (24 hours). Figure 9 shows the average and cumulated perhour production (over the x-axis) and consumption (below the $\mathrm{x}$-axis) of each equipment on the day, for particular load and photovoltaic maximal production curves (and initial conditions).

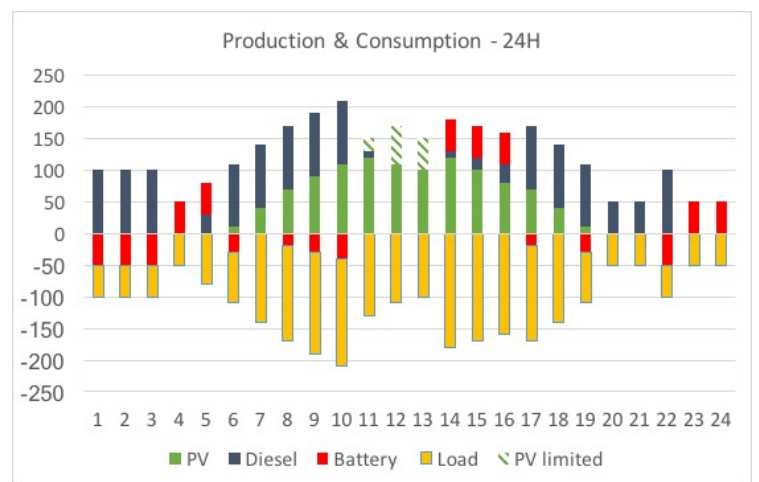

Figure 9: Consumption and production of electricity over a full day

The energy balance has been ensured all day (no black-outs) meaning our design solution is effective on this particular scenario. However, be- tween $11 \mathrm{am}$ and $2 \mathrm{pm}$, the photovoltaic production has been limited (see hatched bars). Looking at the results (not shown in the figure), we see that the charge of the battery was already maximal and could not absorb the extra production. There is consequently a potential for optimization of our solution. Increasing the capacity of the battery, or improving the algorithm of the EMS are two possible iterations. Once the models are updated, the execution of the toolchain automatically updates the simulation artifacts and executes the cosimulation again.

\section{OBSERVATIONS}

The key motivation behind our work is to reduce the cost of iterations in the design of systems by automating the cosimulation of the models using a model-driven approach. To be useful in an industrial context, we need to fulfill the following requirements: each iterative step of the process must provide a quick feedback; the upfront modeling cost must be recovered in the following phases of analysis, maintenance, etc.; business experts must concentrate on their core skills.

We presented in this paper a toolchain based on a cosimulation DSL to reference simulation models and characterize some coupling constraints between them. The various generators allow the generation of simulation units and deployment scripts from this cosimulation model. Hence, this automated process provides the possibility to make changes to the cosimulation scenario with minimal efforts. We illustrate this through the following industrial scenarios.

In a first scenario, a functional architect has to compare components from various vendors, for example to find the best EMS solution (EMSGrid in our previous use case). To guarantee the correct integration of the simulation model provided by the vendor, the tender documents include requirements deduced from the CosiML model. The selection of the right component is simplified because:

- Using the tool chain, the architect can quickly build a test environment, by providing input data inside a CSV file, automatically generating a new FMU and a cosimulation model, then testing multiple configurations easily.

- To select the components to be used in the cosimulation, only the pathFMU attribute of the ProvidedSimulationUnit must be modified 
and the new cosimulation set up can be generated.

In a second scenario, we want to involve electrotechnical engineers to build a load flow model of the power grid (GridFMI in our previous use case). This is possible without an intensive training because they can use their own specific tools to build the simulation model (Dymola, PowerFactory, etc.), and there are only few basic concepts (input, output, discrete or continuous variability) to be explained in order to build the CosiML model. Once they develop a model conforming to the CosiML metamodel, they can then use an iterative approach to improve the model without involving other collaborators, thanks to our toolchain, which automatically integrates their work to the cosimulation platform.

Finally, in a third scenario, we consider the case of a modification of one simulation model inducing a modification of the CosiML model, and especially among the coupling constraints between models (e.g. adding or renaming several ports).Firstly, the validation rules of our toolchain guarantee the consistency of the CosiML model. Secondly, the automated execution process of the cosimulation will raise errors until each impacted simulation model makes the necessary adjustments. Thirdly and finally, the implementation of the adjustments might be partially done by the generators of the toolchain.

\section{CONCLUSION AND PERSPECTIVES}

By automating some verifications and the generation of cosimulation artifacts, model driven approaches allow shorter, less costly and less error prone iterations on a solution design. Our toolchain relies on an abstract CosiML model of the system to check the consistency of the different simulation units, to generate adapters for discrete event signals that cannot be used as is in an FMI simulation, and to generate FMUs from models developed with different tools. It uses the FMI standard and benefits from its many advantages regarding CPS simulation in industry. It can also integrate FMUs exported by some modeling tools in the cosimulation, allowing models from different system domains to be developed with the relevant tools, by experimented teams, while protecting industrial property inside FMUs.

The CosiML language and its generators have been used on a real industrial case, which involves both continuous and discrete signal exchanges. We recently managed to integrate an FMU built from an OMNeT model of a communication network in a cosimulation, which will allow us to run finer simulations of a smart grid that take into account the performance and limitations of the communication network. CosiML and our tool chain have been designed to support new modeling tools and new generators, and the integration of models from OMNeT in the tool chain will be an opportunity to check this. The modular nature of the different transformations also helps to adapt the generated artifacts to different versions of FMI. For instance, the support for a more precise detection of discontinuities in FMI v2.1 may lead to a new adapter for discrete event signals, while keeping the current one for cosimulations using older versions of FMI.

Finally, we want to bring a better integration with the design process of a system using simulation. Indeed, we are aware that developing a CosiML model implies a previous analysis phase, to identify which kind of simulation models need to be developed, and for which purpose. We propose to use another language to represent the system functions to simulate, independently of their implementation models, and to build a model transformation toward CosiML.

\section{REFERENCES}

Andrén, F., Strasser, T., and Kastner, W. (2017). Engineering Smart Grids: Applying ModelDriven Development from Use Case Design to Deployment. Energies, 10(3):374.

Blochwitz, T., Otter, M., Arnold, M., Bausch, C., Elmqvist, H., et al. (2011). The functional mockup interface for tool independent exchange of simulation models. In Proceedings of the 8th International Modelica Conference, pages 105-114.

Broman, D., Greenberg, L., Lee, E. A., Masin, M., Tripakis, S., and Wetter, M. (2015). Requirements for Hybrid Cosimulation Standards. In Proceedings of the 18th International Conference on Hybrid Systems: Computation and Control, HSCC '15, pages 179188. ACM.

Cremona, F., Lohstroh, M., Broman, D., Lee, E. A., Masin, M., and Tripakis, S. (2019). Hybrid co-simulation: it's about time. Software and System Modeling, 18(3):1655-1679. 
Dahmann, J. S. and Morse, K. L. (1998). High Level Architecture for simulation: An update. In Proceedings. 2nd International Workshop on Distributed Interactive Simulation and Real-Time Applications (Cat. No.98EX191), pages 32-40.

Elsheikh, A., Awais, M. U., Widl, E., and Palensky, P. (2013). Modelica-enabled rapid prototyping of cyber-physical energy systems via the functional mockup interface. pages 1-6. IEEE.

Évora Gómez, J., Hernández Cabrera, J. J., Tavella, J.-P., Vialle, S., Kremers, E., and Frayssinet, L. (2019). Daccosim NG: Cosimulation made simpler and faster. In The 13th International Modelica Conference, pages 785-794.

Gomes, C., Meyers, B., Denil, J., Thule, C., Lausdahl, K., Vangheluwe, H., and De Meulenaere, P. (2018a). Semantic adaptation for FMI co-simulation with hierarchical simulators. SIMULATION.

Gomes, C., Thule, C., Larsen, P. G., and Vangheluwe, H. (2018b). Co-Simulation: A Survey. ACM Computing Surveys, 51(3):49:1-49:33.

Guermazi, S., Tatibouet, J., Cuccuru, A., Dhouib, S., Gérard, S., and Seidewitz, E. (2015). Executable modeling with fUML and alf in papyrus: Tooling and experiments. In EXE@MoDELS.

Li, W., Monti, A., Luo, M., and Dougal, R. A. (2011). VPNET: A co-simulation framework for analyzing communication channel effects on power systems. In 2011 IEEE Electric Ship Technologies Symposium, pages 143149.

Muller, S. C., Georg, H., Nutaro, J. J., Widl, E., Deng, Y., Palensky, P., Awais, M. U., and al. (2018). Interfacing Power System and ICT Simulators: Challenges, State-ofthe-Art, and Case Studies. IEEE Trans. Smart Grid, 9(1):14-24.

Nutaro, J. (2011). Designing power system simulators for the smart grid: Combining controls, communications, and electromechanical dynamics. In 2011 IEEE Power and Energy Society General Meeting, pages $1-5$.

Oudart, D., Cantenot, J., Boulanger, F., and Chabridon, S. (2019). An Approach to Design Smart Grids and Their IT System by Cosimulation:. In MODELSWARD 19, pages 370-377. SCITEPRESS - Science and Technology Publications.

Palensky, P., Van Der Meer, A. A., Lopez, C. D., Joseph, A., and Pan, K. (2017). Cosimulation of Intelligent Power Systems: Fundamentals, Software Architecture, Numerics, and Coupling. IEEE Industrial Electronics Magazine, 11(1):34-50.

Paris, T., Ciarletta, L., and Chevrier, V. (2017). Designing co-simulation with multi-agent tools: a case study with NetLogo. In Francesco Belardinelli, E. A., editor, 15th European Conference on Multi-Agent Systems (EUMAS 2017), volume 10767 of MultiAgent Systems and Agreement Technologies, pages 253-267, Évry, France. Springer.

Rohjans, S., Lehnhoff, S., Schütte, S., Andrén, F., and Strasser, T. (2014). Requirements for Smart Grid simulation tools. In 2014 IEEE 23rd International Symposium on Industrial Electronics (ISIE), pages 1730-1736.

Suri, K., Cuccuru, A., Cadavid, J., Gerard, S., Gaaloul, W., and Tata, S. (2017). Modelbased Development of Modular Complex Systems for Accomplishing System Integration for Industry 4.0. In Proceedings of the 5th International Conference on ModelDriven Engineering and Software Development - Volume 1: MODELSWARD, pages 487-495. ScitePress.

Tavella, J.-P., Caujolle, M., Vialle, S., and al. (2016). Toward an Accurate and Fast Hybrid Multi-Simulation with the FMI-CS Standard. In Emerging Technologies and Factory Automation (ETFA-2016), Berlin, Germany.

Van Acker, B., Denil, J., Vangheluwe, H., and De Meulenaere, P. (2015). Generation of an optimised master algorithm for fmi cosimulation. In DEVS Integrative M\&S Symposium, DEVS '15. Society for Computer Simulation International.

Yang, C.-H., Zhabelova, G., Yang, C.-W., and Vyatkin, V. (2013). Cosimulation Environment for Event-Driven Distributed Controls of Smart Grid. IEEE Trans. Industrial Informatics, 9(3):1423-1435.

Zhao, H., Apvrille, L., and Mallet, F. (2017). Multi-View Design for Cyber-Physical Systems. In PhD Symposium at 13th International Conference on ICT in Education, Research, and Industrial Applications, pages 22-28, Kiev, Ukraine. 